\title{
Development of Inplantable Surface Acoustic Wave Sensor for High Voltage Cable Core Temperature Monitoring in Intermediate Joint
}

\author{
Dan Pang ${ }^{1,}{ }^{*}$, Xiaoyan Wang ${ }^{1}$, Changpeng Zhao $^{1}$ and Chaobin Wang ${ }^{2}$ \\ ${ }^{1}$ State Grid Changchun Power Supply Corporation, 130021 Changchun, China \\ ${ }^{2}$ Eletrical Engineering, Northest electric power university, 132012 Jilin, China
}

\begin{abstract}
In this paper, a kind of implantble passive wireless surface acoustic wave (SAW) sensor and its reader are developed to measure the operation temperature of high voltage crossed-linked cable core. A small type SAW temperature sensor is embedded into cable intermediate joint. A radio frequecny transceiver of the reader based on discrete devices is optimally designed to achieve high signal-to-noise ratio and receiving sensitivity. The characteristic of resonant frequency and temperature of SAW sensor is determined by frequency scan method. Through AC withstand voltage and temperature rise test of $110 \mathrm{kV}$ cable, it proves that the prototype can accurately measure the operation temperature of $110 \mathrm{kV}$ cable core in intermediate joint under high operation temperature and strong electromagnetic environment.
\end{abstract}

\section{Introduction}

In recent years, a number of cable explosion and fire accidents in cable tunnel have occurred in urban power distribution networks in China[1], which has caused large scale blackout in urban areas and huge economic loss to power supply companies and users. The main cause of cable accidents is the overheat of the cross-linked cable joint, for which insulation aging is accelerated. With miles of cable lines in operation increasing, the number of crosslinked cable intermediate joints becomes huge. Due to the installation process or manufacturing quality substandard, high line operating temperature and other reasons, the cross-linked cable joint heating has become a hidden danger that threatens the safe operation of power grid.

The operation temperature of cable core is an important parameter to evaluate the operation state of cable line. There are two ways of cable tempera-ture measurement: indirect skin type and direct core type. Due to the complexity of cable insulation structure, indirect temperature measurement technologies based on active wireless sensor, infrared thermal image or distributed optical fiber are widely applied[2-3]. However, the measured skin temperature and cable line current should be input into the dynamic calculation model of cable core temperature to improve the accuracy. A passive wireless temperature sensor is developed embedded in the insulation layer of power cable by using an ultra-high frequency RFID temperature sensor chip[4]. There are some disadvantages: the antenna and the sensor are not packaged together, which may be damaged by external force; the active temperature sensor chip is easily interfered by the strong physical field of the high-voltage cable. Reference[5] proposes a temperature measurement method based on passive RLC network of measuring the damping coefficient of response signal, but with low accuracy and stability.

The passive wireless surface acoustic wave (SAW) sensor is especially suitable for high temperature, strong electromagnetic interference, surrounding metal and other harsh environment applications and widely used in medical[6], chemical, and industrial fields. References[79] have successfully applied SAW sensor to the surface temperature monitoring of power equipment such as substations, high-voltage switches, and power cables.

In this paper, a kind of passive wireless SAW temperature sensor embedded into cable intermediate joint and its reader are developed. The structure is as follows. Section II briefly introduces the principle of resonant SAW temperature sensor using the frequency scan method. In Section III, the structure of cable core SAW temperature sensor and the radio frequency(RF) transceiver of reader are optimally designed to realize the stable wireless communication. Section IV presents AC withstand voltage and temperature rise test results of prototype installed in a section of $110 \mathrm{kV}$ cable intermediate joint. The conclusion of whole paper is summarized in Section V.

\section{Princeple of SAW temperature sensor}

Now, there are mainly two types of SAW sensors: delayline type and resonant type. Compared with delay-line type, resonant type SAW sensor has advantages of high quality factor, small insertion loss, good frequency stability, strong anti-interference ability, and high accuracy, which is more suitable for sensing applications. However, 
the delay line type SAW sensor is mainly used for application of electronic label.

Fig. 1 shows the principle of resonant type SAW temperature measurement. It depends on near filed wireless communication between SAW sensor and its reader. In Fig.1(a), the reader firstly sends out excitation signal that is a narrow pulse signal with pulse width of $40 \mu \mathrm{s}$ and sinusoidal carrier wave frequency band of $433 \mathrm{MHz}$ in Fig. 1(b). The sensor antenna is connected to inter-digital transducer inside SAW resonator, and the conversion between electromagnetic waves and surface acoustic waves is completed through piezoelectric and inverse piezoelectric effects. The surface acoustic waves are reflected and superimposed back and forth between reflection grids on both sides. Resonance occurs when the frequency of sinusoidal carrier is the same as natural frequency of SAW resonator. The SAW resonator can be equivalent to an RLC series circuit. Due to intermittent excitation, the response signal of Fig. 1(b) presents a waveform of attenuated oscillation with resonant frequency as fundamental frequency component.

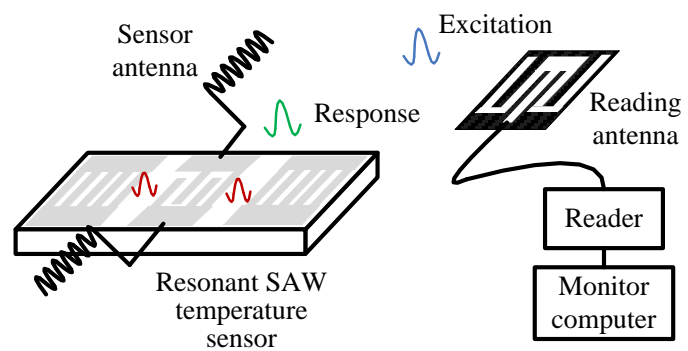

(a)SAW temperature measurement system

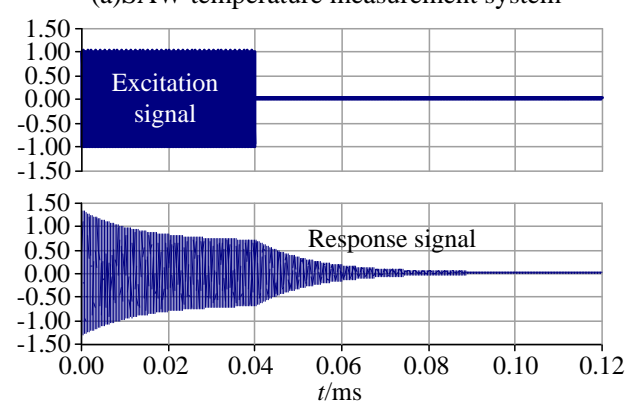

(b)Excitation and response signal of SAW resonator

Fig. 1. The principle of SAW temperature sensor

The resonant frequency of SAW resonator is influenced by temperature. Assumed the resonance frequency is $f_{0}$ at $T_{0}$, the relationship between resonant frequency $(f)$-temperature $(T)$ can be approximated by a third-order polynomial, and written as

$$
f=f_{0}\left[1+a_{0}\left(T-T_{0}\right)+b_{0}\left(T-T_{0}\right)^{2}+c_{0}\left(T-T_{0}\right)^{3}\right]
$$

Where coefficients of $a_{0}, b_{0}$, and $c_{0}$ can be easily determined by frequency scan experiment at different temperature. According to the characteristic of the maximum response signal strength corresponding to the resonant frequency, the frequency scan is carried out by transmitting excitation signal with the same power strength and the carrier frequency increasing at a setting step $(\Delta f)$ in the range of $\left[f_{\min }, f_{\max }\right]$. Then the corresponding response signal strength indicator (RSSI) is detected and the resonant frequency is determined by finding out the maximum RSSI.

Fig. 2 shows the frequency scan results of a SAW sensor at $63.8^{\circ} \mathrm{C}$ and $\Delta f$ of $25 \mathrm{kHz}$ in the range of [427.95,429.525] MHz. The temperature-resonant frequency characteristic of SAW sensor in Fig.3 shows good linearity.

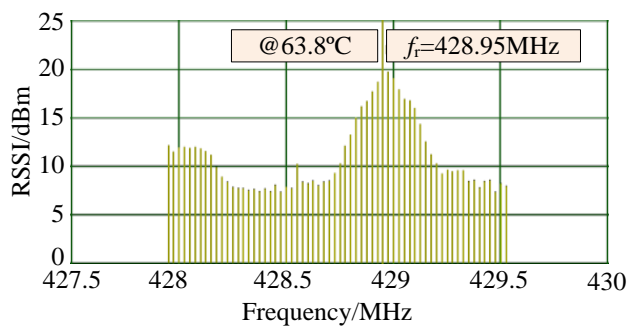

Fig. 2. The frequency scan results of a SAW sensor.

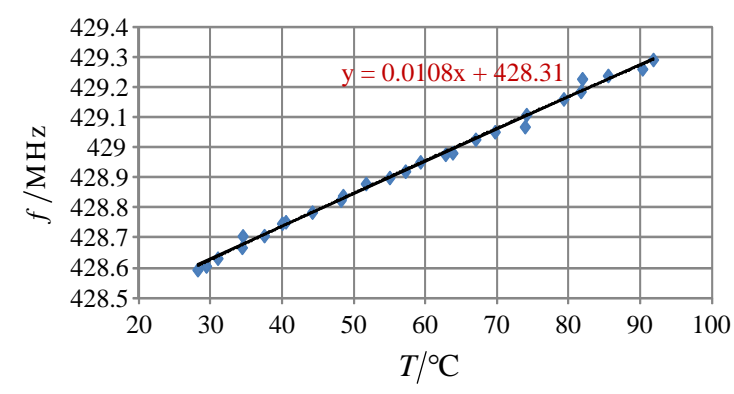

Fig.3. The characteristic of temperature and resonant frequency of SAW sensor

\section{Design of SAW sensor and reader}

When a wireless SAW sensor embedded into a cable intermediate joint, the problem that signal strength becomes weak after penetrating through insulation layer and metal shield layer is to be solved. The RF circuit of SAW sensor and its reader are designed optimally to ensure good wireless communication.

\subsection{The cable core SAW sensor design}

Fig.4 shows the ring structure of SAW sensor sheathed on the cable core and close to the connector in $110 \mathrm{kV}$ cable intermediate joint. The sensor shell has an outer diameter of $64 \mathrm{~mm}$, an inner diameter of $32 \mathrm{~mm}$, and a thickness of $16 \mathrm{~mm}$, which matches the cross-sectional area of $110 \mathrm{kV}$ cable core and the thickness of main insulation layer.

As shown in Fig.4(a), SAW temperature sensor consists of $433 \mathrm{MHz}$ helical dipole flexible antenna, SAW resonator and heat conducting copper sheet. The size of sensor is small enough to be packaged in the groove of ring shell. The helical dipole antenna radiation has high gain in the direction of cable core, which makes it possible to receive and radiate ectromagnetic wave well. The copper sheet welded on a copper pad at the back of SAW resonator, and connects one pole of the dipole antenna with cable core. On the one hand, the heat of cable core can be quickly transferred to SAW resonator. On the other hand, the cable core can work as a part of the sensor 
antenna, which means the coupling effect between cable core and reading antenna can greatly improve the signal strength of SAW sensor.

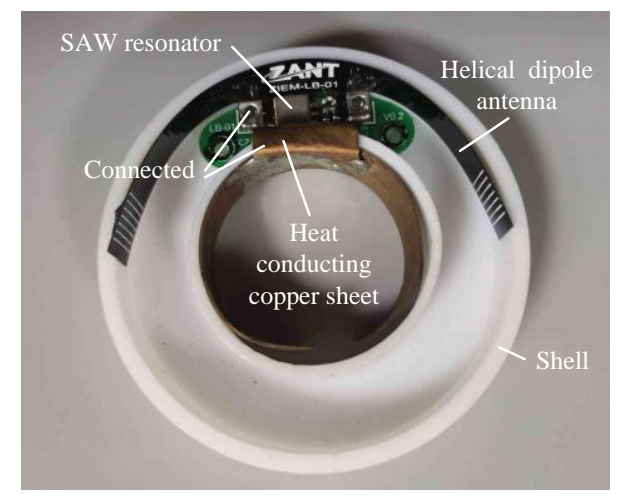

(a)The structure of SAW temperature sensor

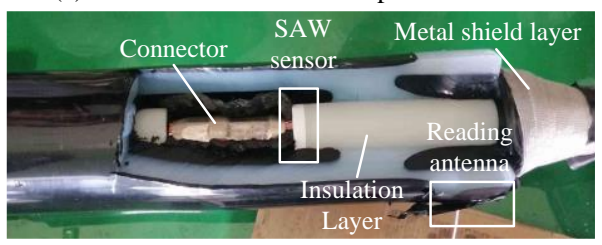

(b) $110 \mathrm{kV}$ cable intermediate joint

Fig. 4. The SAW sensor structure of $110 \mathrm{kV}$ cable core

\subsection{The RF transceiver design of reader}

The controller of reader adopts the digital signal processor(DSP) of TMS320F28335 in interaction with monitor computer and SAW sensor respectively. Determined by the equivalent RLC circuit parameters of SAW resonator, the response signal attenuation is so fast, generally tens of microseconds, that the RF transceiver of reader uses discrete devices to achieve wireless communication. Fig.5 shows the block diagram of transceiver, including programmable frequency synthesizer, transmitter, receiver, RF switch and antenna. Passive wireless SAW sensor is sourced from the reader. For high sensitivity detection of response signal, the transceiver design should meet requirements that the transmitter can provide sufficient transmitting power, while the receiver has high signal-to-noise ratio (SNR) and receiving sensitivity [10].

The frequency synthesizer adopts the chip of $\mathrm{Si} 4112$ integrated with programmable VCO+PLL. Si4112 is controlled by DSP to generate two analog pure sine signal sources at frequency band of $433 \mathrm{MHz}$, served as the local oscillators for the excitation signal modulation and the received signal demodulation respectively. The frequency difference between two oscillators is $10.7 \mathrm{MHz}$ that is the output frequency of the receiver intermediate frequency (IF) subsystem of AD608.

To provide sufficient transmitting power and avoid excessive power accelerating the aging of transmitter, the transmitter consists of an attenuator, a low noise amplifier (LNA) and a general power amplifier (PA). The DSP adjusts the attenuation gain of the attenuator according to the RSSI of the response signal. The first-stage amplifier adopts the high performance LNA chip of RF2373 to ensure its output has a higher signal-to-noise ratio and reduce the impact of noise on the secondary system. The second stage is amplified by a internal PA of the RF switch chip of RF6504, achieving a maximum transmitting power to $1 \mathrm{~W}$. The transmitter is also in cooperation with a highgain dipole antenna.

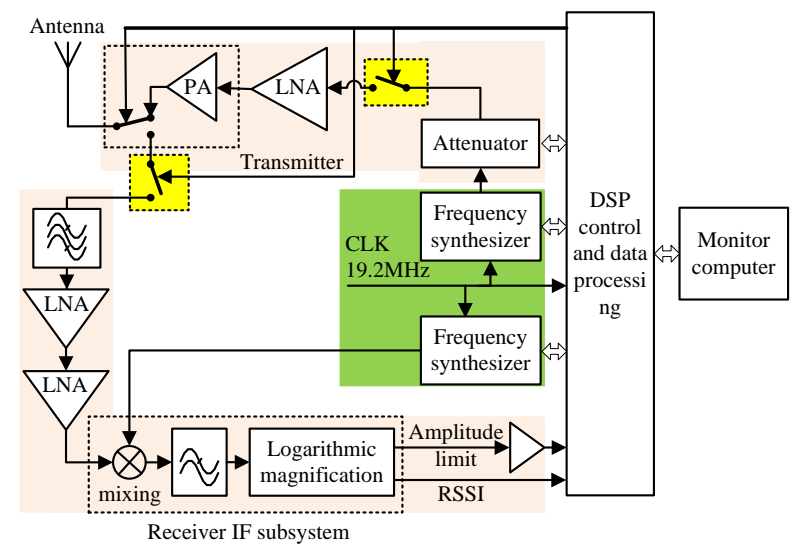

Fig.5. The RF circuit block diagram of reader

To improve the SNR and receiving sensitivity, the receiver consists of a band-pass filter, a two-stage LNA and IF subsystem. In the receiving mode, received signal is filtered by a SAW band-pass filter, where only the 433 MHz frequency band signal is reserved. The filtered signal is weak and amplified by a two-stage LNA amplifier with the $20 \mathrm{~dB}$ gain of each stage. Finally, AD608 processes the amplified signal with mixing, lowpass filtering and converting into a IF signal of $10.7 \mathrm{MHz}$. By logarithmic amplification, it outputs RSSI signal that can be sampled by on-chip AD module of the DSP.

In addition, to reduce the influence of high-frequency noise such as transmitters and frequency synthesizers on the receiver, the following measures are taken. One is to set up a high-isolation single-pole single-throw RF switch chip of ADG901 on the signal path of the transmitter and receiver of Fig. 4, effectively suppressing the interference that propagates along the "path". Another is to keep the receiver away from the transmitter and the frequency synthesizer and cover a metal shield during the PCB layout, thereby effectively suppressing the interference that propagates along the "field".

\section{Prototype test}

Fig. 6 shows a $110 \mathrm{kV}$ cable high voltage and high current test platform to evaluate temperature measurement performance of implantable SAW sensor. The SAW temperature sensor is installed in the intermediate joint and close to connector, and reading antenna is installed on the surface of joint insulating bushing in Fig.6(b). The distance between the sensor and reading attenna is about $20 \mathrm{~cm}$.

To simulate temperature rise test of cable core, two ends of tested cable in Fig.6(a) should be short-circuited with a copper bar, and high current generator generates high cable current through two CTs to heat the cable core. The XZL-type series resonance high votage test device generates $50 \mathrm{~Hz} \mathrm{AC}$ high voltage to act on the cable core 
to simulate the cable $\mathrm{AC}$ withstand voltage test.

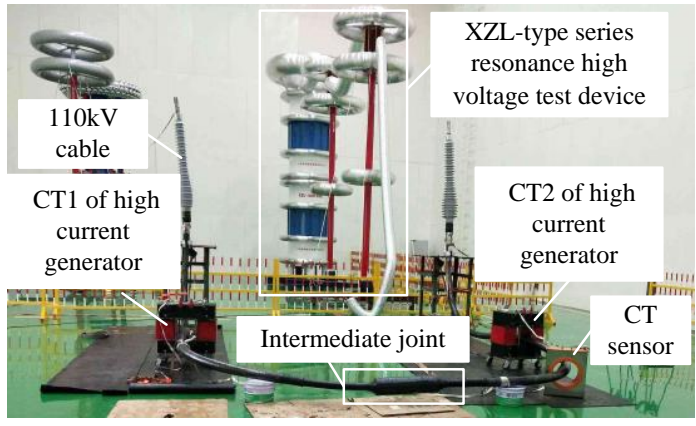

(a) High voltage and high current test platform
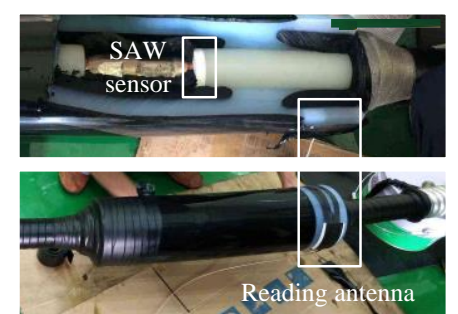

(b)The installation position of SAW sensor and reading antenna

Fig. 6. The high voltage and high current test platform of $110 \mathrm{kV}$ cable

\subsection{Temperatue rise test}

Fig. 7 shows the result of temperature rise test. At the stage one of test, the cable core is preheated about $1 \mathrm{~h}$, and then increases cable current for rapid heating. During the period of heating, the monitor computer records SAW temperature data every 20 seconds. The RSSI value of Fig.7 remains stable during the temperature rise, and the average is nearly $8 \mathrm{dBm}$. The temperature curve reflects the temperature rise law of cable core. The cable core temperature slowly rises from $20^{\circ} \mathrm{C}$ to $42^{\circ} \mathrm{C}$ in the constant current mode during stage one. In the second stage, the cable current is reduced and maintains ten minutes, during which the core temperature begins to slowly drop. In the third stage, the core current is increased and the temperature rises quickly as cable current increases to level of $\mathrm{kA}$.

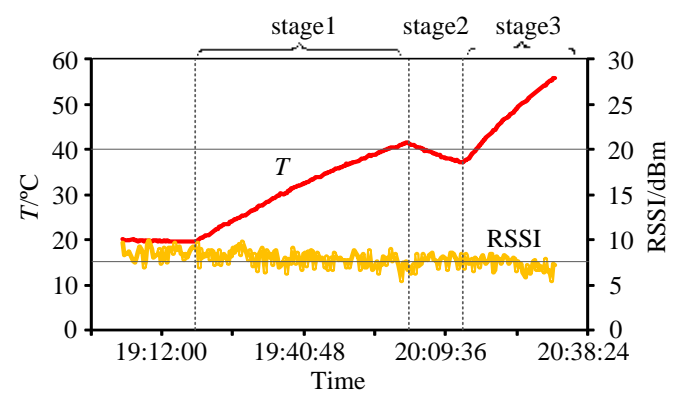

Fig. 7. The result of temperature rise test

In addition, a thermocouple thermometer TES1310 and a mesh probe NR81531B are used to measure the temperature of the short-circuited copper bar of cable to vadiate the accuracy of SAW temperature measurement results. Obiviously, the measured temperature by two methods in Fig.8 is close. Thus temperature rise test results verfy that the communication between SAW sensor and reader is stable, and the measured temperature can accurately reflect the cable core operation temperature.

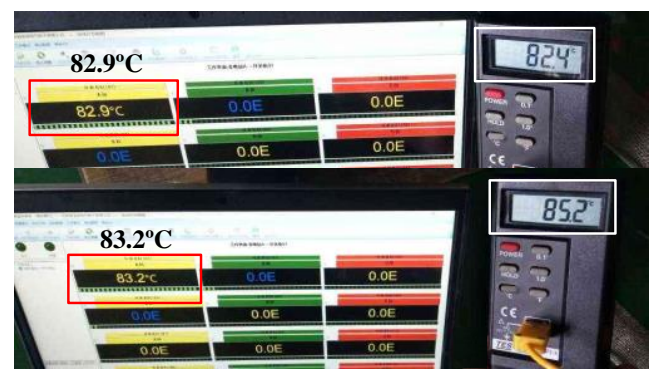

Fig. 8. The comparison of temperature measured by SAW sensor and Thermocouple

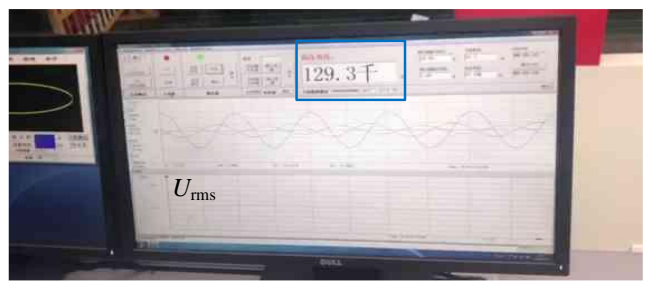

(a) AC test voltage

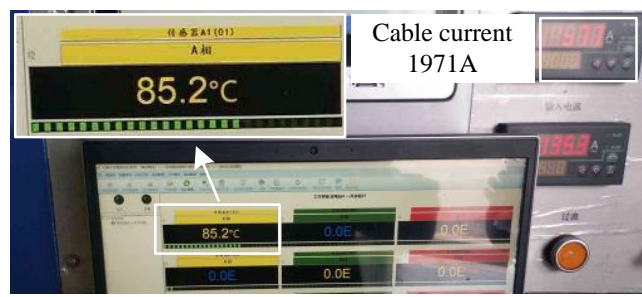

(b) Temperature and RSSI measured at 8:48

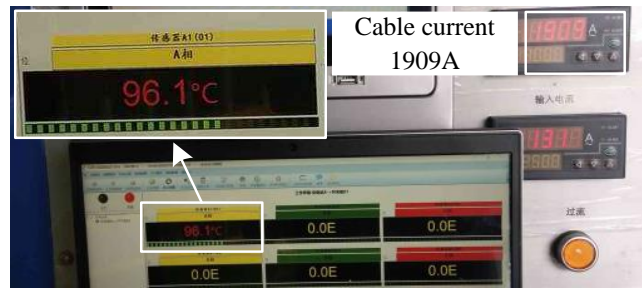

Temperature and RSSI measured at 9:23

Fig.9. The result of ac voltage withstand and high current test

\subsection{High voltage and high current test}

High voltage and high current are simultaneously acted on the cable core to evaluate the feasibility of implantble SAW sensor under strong electromagnetic field and high operation temperature condition. As shown in Fig.9(a), the present AC test voltage is $129.3 \mathrm{kV}$, twice as much as the rated operation phase voltage of $110 \mathrm{kV}$ cable. The operation cable current exceeds 1.9kA in Fig.9(b)-(c). As the window of SAW measurement results enlarged, it shows that the RSSI bar indicates a medium strength. When the temperature exceeds $90^{\circ} \mathrm{C}$ that is the upper limit of allowable operation temperature, the colour of temperature value turns red and raise alarm. From Fig.9(b) and Fig.9(c), the temperature of cable core having risen from $85.2^{\circ} \mathrm{C}$ to $96.1^{\circ} \mathrm{C}$ takes time of 35 minutes. Above all, 
the prototype of SAW sensor maintains good wireless communication and strong anti-interference ability under conditions of continuous high operation temperature and strong electromagnetic environment, thereby achieving online temperature monitoring of cable core in intermediate joint.

\section{Conclusion}

In this paper, an implantable SAW temperature sensor and its reader are developed in the intermediate joint of the high-voltage cross-linked cable. The ring-type SAW sensor is sheathed on cable core close to the connector of intermediate joint, and the cable core temperature is directly sensed by a heat conducting copper sheet. With the coupling effectc of cable core, the signal strength of SAW sensor is greatly enhanced. By improving the transmitting power and receiving sensitivity of reader, the stability of wireless communication is ensured. The AC withstand voltage and temperature rise test results of a section of $110 \mathrm{kV}$ cable prove that the prototype has strong anti-interference ability and high temperatue accuracy under conditions of high operation temperature and strong electromagnetic interference environment, and can raise an over-temperature alarm in time.

\section{References}

1. Fenglian Liu, Yuanshi deng, Zhihang Xue, et al. Electric Wire \& Cable, (6): 36-40(2017)

2. Yunpeng Gao, Tianyuan Tan, Kaipei Liu. Insulating Materials, 47(6): 13-17(2014)

3. Hua Jiang, Xizhong Li, Xiaolai Qin et al. Instrument Technique and Sensor, (12): 58-62(2018)

4. Xiang $\mathrm{Wu}$, Yigang He, Fangming Deng, et al. Instrument Technique and Sensor, (6): 113-117(2018)

5. Mengkun Yan, Jiande Wu, Chongwen Zhao, et al. High Voltage Engineering, 39(11): 2664-2669(2013)

6. Xuesong Ye, Xuejun Wang, Bo Liang, et al. Journal of Zhejiang University (Engineering Science), 45(11): 2068-2072 (2011)

7. Zhengjian Zhang, Long Cheng. Modern Electronics Technique, 41(9): 117-120(2018)

8. Xingang Chen, Tang Zhao, Bing Yu, et al. High Voltage Apparatus, 54(8): 48-54(2018)

9. Huhu Yue, Bin Peng, Ling Li, et al. Piezoelectrics \& Acoustooptics, 43(1): 1-4(2021)

10. Ning Yin, Lianbo Zhang, Binghua $\mathrm{Su}$, et al. Instrument Technique and Sensor, (4): 51-54(2018) 